\title{
Equivalent linearization of the Bouc-Wen hysteretic model
}

\author{
Jorge E. Hurtado ${ }^{\text {a }}$, Alex H. Barbat ${ }^{\text {b,* }}$ \\ a National University of Colombia, Apartado 137, Manizales, Colombia \\ b Technical University of Catalonia, c/ Gran Capitán, s/n, Barcelona 08034, Spain
}

Received 8 February 1999; accepted 14 June 1999

\begin{abstract}
The smooth endochronic hysteretic Bouc-Wen model is studied from the point of view of random vibration. The sources of the errors of the method of equivalent linearization applied to this model using the hypothesis of joint Gaussian behaviour are examined. The method of linearization for softening hysteretic models proposed by the authors, which is based on a combination of Dirac and Gauss densities, is developed and applied to the Bouc-Wen model under a variety of conditions. It is shown that the method gives excellent estimations of the response statistics without increasing the computational effort required by the conventional technique. (C) 2000 Elsevier Science Ltd. All rights reserved.
\end{abstract}

Keywords: Random vibration; Hysteretic structures; Equivalent linearization

\section{Introduction}

The method of stochastic equivalent linearization has its roots in the work of Krylov and Bogoliubov [1] on deterministic linearization and it first appeared in papers by Caughey [2], Iwan [3] and others in a probabilistic framework. A great impulse to practical applications of the method was given by a paper of Atalik and Utku [4], who demonstrated that the assumption of a Gaussian behavior of all the state variables greatly simplifies the computation of the linearization co-efficients. Later it was demonstrated that such simplification is valid only for the Gaussian density [5]. Mathematical questions such as the existence and uniqueness of the solutions had already been discussed [6]. A monograph by Roberts and Spanos [7] on computational techniques in nonlinear stochastic dynamics shows that among them this is the method with the clearest appeal to the practical analysis of large structures.

On the other hand the introduction of a smooth and versatile model of hysteresis by Bouc [8] and its posterior development by Wen [9] and others [10-12] opened the way to the application of the method in many

\footnotetext{
* Corresponding author. Tel.: +34-93-401-6496; fax: +34-93-4016517.

E-mail address: barbat@etseccpb.upc.es (A.H. Barbat).
}

structural dynamics fields such as vibration of frames [13], steel and concrete structures [14-16], soil profiles [17], three dimensional frames [18], base isolation [19] and hybrid control [20]. The smooth hysteretic model has been well received in nonlinear stochastic dynamics not only because of its versatility but also for the possibility of calculating the linearization matrices in explicit form. This is not possible for piecewise hysteretic models, such as elasto-plastic, bilinear, origin-oriented, etc. [21-23].

The accuracy of the method has also been investigated. Generally speaking, it can be said that the magnitude of the errors depends on many factors, such as oscillator's type and parameters, type of calculation (i.e., stationary or nonstationary), excitation level, etc [7]. For the specific case of the Bouc-Wen hysteretic model, it has been found that under the assumption of joint Gaussian behaviour, the stationary estimations given by the method are reasonably good but the nonstationary displacement response is commonly underestimated $[10,24]$. The errors can be quite large in some cases. Given the importance of displacement estimations in design, attempts to overcome this situation have been proposed. For instance, Pradlwarter and Schueller [25] recommended the use of a nonlinear transformation of the density of the hysteretic component, together with the application of the Nataf method [26] to estimate the joint density functions necessary for calculating the lin- 
earization coefficients. Since the target density of the hysteretic component is not known beforehand, the realization of some Monte Carlo simulations is proposed to fill this gap. In case of real structural systems the latter device will, of course, largely increase the computational effort. On the other hand, Kimura et al. [23] proposed the use of a truncated Gaussian density combined with Dirac pulses to simulate the effect of the concentration at the maximum values of the hysteretic component of bilinear systems. Mention must also be made of a different, way of upgrading the statistics deriving from the Gaussian assumption, which consists in the use of empirical factors and equations. An important method of this kind is due to Park [27], who proposes the use of some coefficients obtained through intensive Monte Carlo simulations. A similar approach was adopted by Yeh [28] to the specific case of hysteretic spatial buildings modelled as shear beams.

This paper has two main purposes. First, to contribute to the clarification of the somewhat confused scenario of the errors inherent to the conventional Gaussian method of linearization when applied to the Bouc-Wen model. Second, to improve the method proposed by the

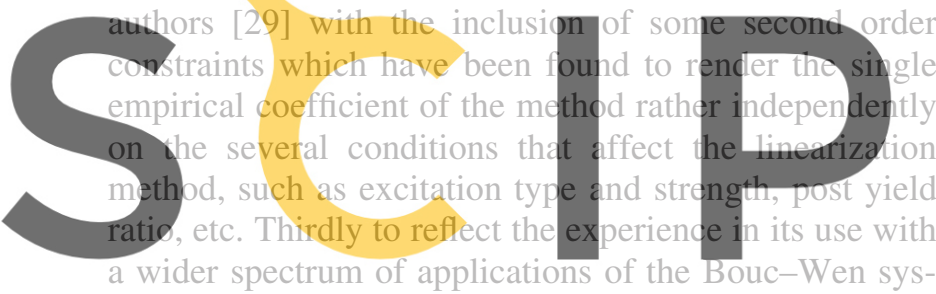
tem than in the original paper [29], in order to demon-

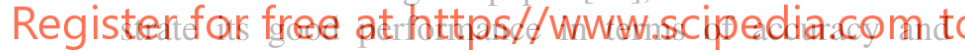
computational labour. The conclusions drawn from this study could be useful for random vibration analyses using extensions of this basic smooth endochronic model.

\section{Gaussian stochastic linearization}

Let us consider a single degree of freedom structure having a restoring force of the Bouc-Wen endochronic type. The equation of motion is

$m \ddot{X}+c \dot{X}+\alpha k X+(1-\alpha) k Z=P(t)$

where the nonlinear random variable $Z$ is the solution of the following differential equation

$\dot{Z}=h(\dot{X}, Z)=A \dot{X}-\beta|\dot{X}||Z|^{n-1} Z-\gamma \dot{X}|Z|^{n}$

An stochastically equivalent linear equation will be looked for in the form

$\dot{Z}=s_{\mathrm{e}} X+c_{\mathrm{e}} \dot{X}+k_{\mathrm{e}} Z$

where $s_{\mathrm{e}}, c_{\mathrm{e}}$ and $k_{\mathrm{e}}$, are coefficients which are calculated by minimizing the expected value of the square error
$\epsilon^{2}=\left[h(\dot{X}, Z)-\left(s_{\mathrm{e}} X+c_{\mathrm{e}} \dot{X}+k_{\mathrm{e}} X\right)\right]^{2}$

Under the hypothesis of joint Gaussian behaviour of the state vector $Q^{\mathrm{T}}=[X, \dot{X}, Z]$ the values of the coefficients are [10]

$s_{\mathrm{e}}=0$

$c_{\mathrm{e}}=E\left[\frac{\partial h}{\partial \dot{X}}\right]=A-\beta F_{1}-\gamma F_{2}$

$k_{\mathrm{e}}=E\left[\frac{\partial h}{\partial Z}\right]=-\beta F_{3}-\gamma F_{4}$

where functions $F_{\mathrm{i}}, i=1,2,3,4$ are given by

$F_{1}=\frac{\sigma_{\mathrm{Z}}^{n}}{\pi} \Gamma\left(\frac{n+2}{2}\right) 2^{n / 2} I_{\mathrm{s}}$

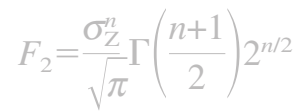

$F_{3}=\frac{n \sigma_{\dot{X}} \sigma_{Z}^{n-1}}{\pi} \Gamma\left(\frac{n+2}{2}\right) 2^{n / 2}\left(2\left(1-\rho_{X Z}^{2}\right)^{\frac{n+1}{2}}+\rho_{\dot{X} Z} I_{s}\right)$

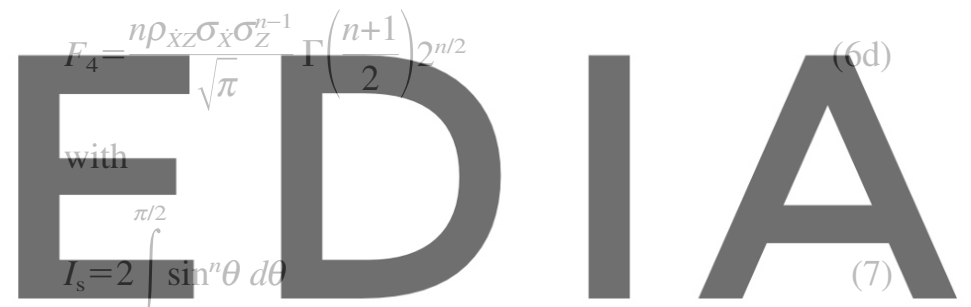

\section{download-the version without the watermark $l=\tan ^{-1}\left(\frac{\rho_{\dot{X} z}}{\rho}\right.$

It must be noted that no closed expressions such as these can be obtained for piecewise hysteretic models, in which case the linearization coefficients are typically expressed through integrals, which in nonstationary analyses should be solved at each time step [7]. The statistics required in the above expressions can be obtained by solving the differential equation for the covariance $\sum(t)=E\left[Q Q^{\mathrm{T}}\right]$

$\sum(t)=A_{\mathrm{e}}(t) \sum(t)+\sum(t) A_{\mathrm{e}}^{\mathrm{T}}(t)+2 \pi S_{\mathrm{F}}(t)$

in which $A_{\mathrm{e}}(t)$ is the equivalent system matrix, which can be assembled after the equilibrium Eqs. (1) and (3) and $S_{\mathrm{F}}(t)$ is the matrix of external modulated white noises. See [7] for details.

\section{Error sources}

It has been pointed out that the above Gaussian linearization of the Bouc-Wen model leads in some cases 
to reasonably good estimations of the second order response when compared with the results of Monte Carlo simulation. Such is the case, for example, of stationary response estimations of structures subject to filtered or unfiltered white noise $[7,10]$. In other instances, however, large discrepancies can take place $[24,25]$. The errors concern mainly the nonstationary estimation of displacement statistics which is one of the most important variables in practical design. The standard deviation of the velocity and hysteretic force, as well as the covariances are reasonably well estimated. The following is a description of the error sources in the estimation of displacement statistics based on the published literature on the subject as well as on the authors' experience.

\subsection{Probability density assumption}

The most evident cause of error lies in the assumption of Gaussianity of all the state variables. It has been shown that while Gaussianity is reasonably admissible for displacement and velocity, as far as only second order information is concerned, it is clearly inadequate

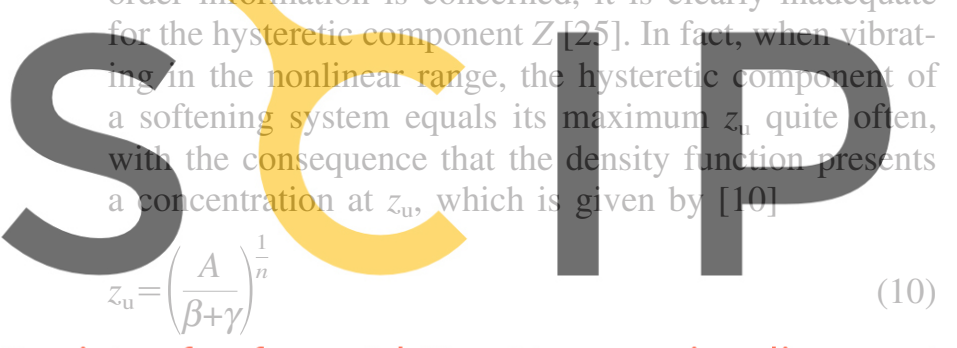

Register for free at https//www.scipedia.com to

\subsection{Drift motion and excitation model}

A second cause of errors lies in the tendency of some hysteretic systems to drift, that is, to loose the central position as the mean value of the vibration [24]. In a force-displacement diagram, this is reflected as an errance of the hysteresis loops' center. Note that, this is a genuine nonlinear phenomenon which is difficult to reproduce with an equivalent linear system. In the frequency domain the driving drift motion can be regarded as a very low frequency wave which in the power spectral density of the displacement response appears as a large peak. For hysteretic models this phenomenon is the stronger, the lower the value of $\alpha$ [27]. However, the magnitude of the errors of the Gaussian method brought about by drift is closely related to the excitation spectrum. In fact, for purely white noise excitation, low frequency waves tend to produce drift irrespective of the hardening ratio $\alpha$. Nevertheless: the drift is somewhat reproduced by the Gaussian method under white excitation. This can be atributed to the assymptotic values of the displacement transfer function. For an equivalent linear system with natural frequency $\bar{\omega}$ it is given by
$\left|H_{\mathrm{X}}(i \omega)^{W}\right|^{2}=$

$$
\overline{\left(-\omega^{2}+\alpha \bar{\omega}^{2}+\frac{(1-\alpha) \bar{\omega}^{2} \omega^{2} c_{\mathrm{e}}}{\omega^{2}+k_{\mathrm{e}}^{2}}\right)^{2}+\left(2 \bar{v} \bar{\omega} \omega-\frac{(1-\alpha) \bar{\omega}^{2} \omega c_{\mathrm{e}} k_{\mathrm{e}}}{\omega^{2}+k_{\mathrm{e}}^{2}}\right)^{2}}
$$

Notice that it tends to $1 / \alpha^{2} \bar{\omega}^{4}$ as $\omega \rightarrow 0$. This renders the equivalent system able to produce some low frequency responses. In contrast, when low-cut filters are introduced (as in the Clough-Penzien [30] or Iwan-Paparizos [24] seismic models) the Gaussian method becomes unable to reproduce the system's drift because the transfer function suppresses the low frequency responses. In fact, in the Clough-Penzien case the modulus is given by

$\left|H_{\mathrm{X}}(i \omega)^{F}\right|^{2}=\left|H_{\mathrm{X}}^{W}(i \omega)\right|^{2}\left|H^{C P}(i \omega)\right|^{2}$

with
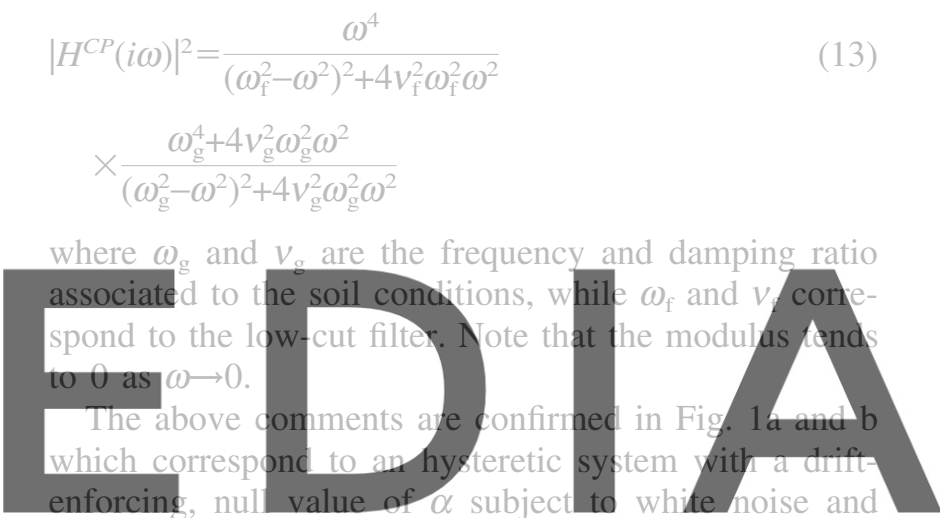

Clough-Penzien excitations, respectively. It can be seen

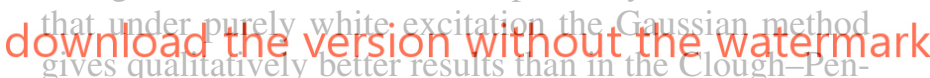
zien case, for which the method does not follow the displacement trace after yielding. When a modulating function is introduced in connection to the Clough-Penzien filter as a realistic nonstationary model of seismic action, the errors can be as large as 300 percent, as Fig. 2 shows. The modulating function employed in this case is that of Shinozuka and Sato [31] given by

$\xi(t)=\frac{1}{c}\left(\mathrm{e}^{-a t}-\mathrm{e}^{-b t}\right)$

with

$c=\max \left(\mathrm{e}^{-a t}-\mathrm{e}^{-b t}\right)=\left[\left(\frac{a}{b}\right)^{\frac{a}{b-a}}-\left(\frac{a}{b}\right)^{\frac{b}{b-a}}\right]$

The parameters $a=0.085$ and $b=0.17$ have been used, which correspond to a long duration earthquake. Notice that in cases like this the Gaussian method not only fails in capturing the peak value but also in assessing the correct shape of the response. As a matter of fact, since the Gaussian method is unable to trace the drift errance, it becomes entirely governed by the modulating function. Note that for many materials, such as reinforced concrete, steel [15] or soil [16] very low values of $\alpha$ have 
(a)

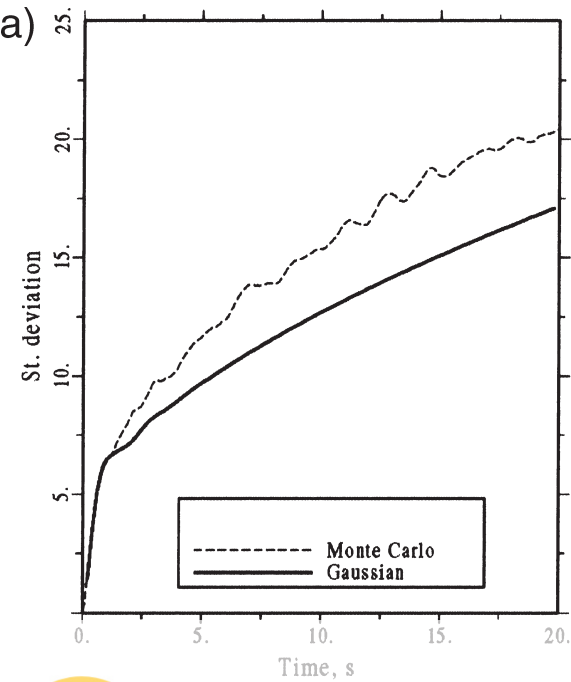

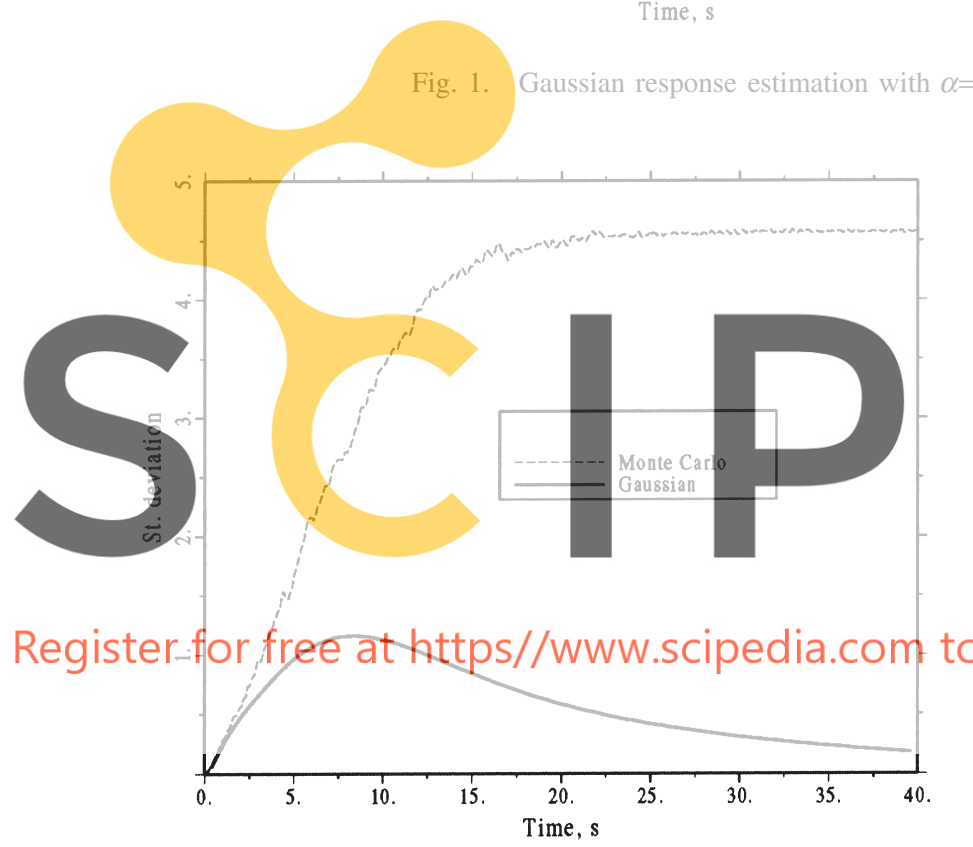

Fig. 2. Gaussian response with modulated Clough-Penzien load $(\alpha=0)$.

been proposed, so that some amount of drift can be expected in their response. As for nonstationary analyses the reliability assessment must be based on the whole evolution of the level crossing rate [32], the importance of estimating correctly the whole evolution of the response moments needs not to be emphasized.

\subsection{Nonlinearity degree of the response}

A third cause of errors is the nonlinearity degree of the response. In fact, for very low or very large excitation levels (as measured by the power spectral density function) the response of the hysteretic system is clearly a narrow band, because the structure responses are either in the linear or in the nonlinear ranges. When subject to a middle strength excitation the response shows a larger

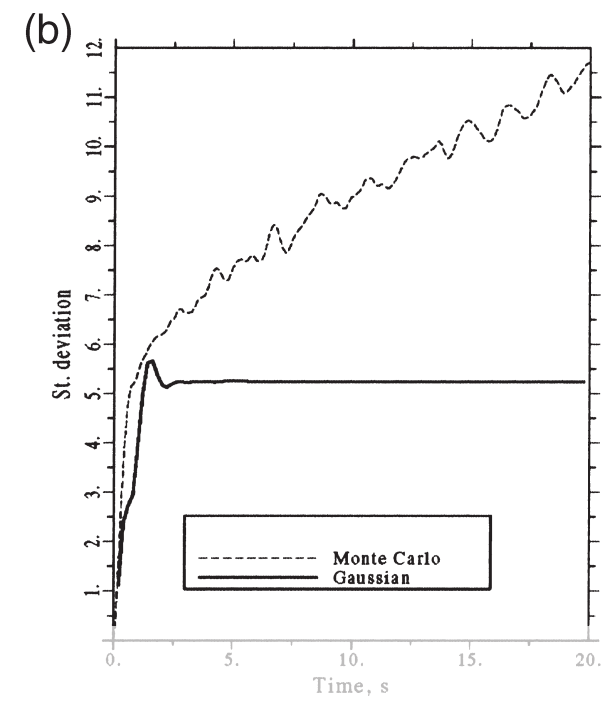

=0. (a) White noise; (b) Clough-Penzien spectrum.

width [7]. By means of stationary analyses that scan the physically relevant intensity range of white excitation it has been demonstrated, however, that this kind of error affects the response estimations of piecewise rather than
those of smooth hysteretic models [7,10]. Howgver, in
nonstationary analyses of the latter, such errors appear
and, generally speaking, they are larger, the ligher the
nonlinearity degree of the response, as will be shown in
the numerical examples at the end of the papel.
For explaining the diverse accurdey of stationary and nonstationary calculations in this respect it must be taken

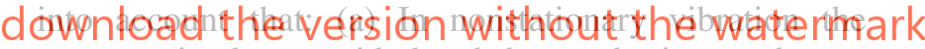
response is always wide-band due to the imposed transition from linear to nonlinear states; (b) Their numerical procedures are rather different, the former proceeding by time-independent iteration [11] and the latter by solving a system of differential equations in which the response at any instant depends on the whole estimated evolution. Accordingly, one could regard the nonstationary problem as one more depending on the assumption of Gaussianity than the stationary one. Since Gaussianity means linearity in this context, due to the fact that the response of a linear system to Gaussian input is always Gaussian, it is evident that when the structure starts the departure from linearity in the nonstationary evolution it also departs from Gaussianity and thus the accuracy of future estimations under such hypothesis will depend on the current degree of response nonlinearity.

\subsection{Loop shape}

Last but not least, the shape of the hysteretic loops, as defined by the model parameters, has an important influence on the errors of the Gaussian method. In the Bouc-Wen model softening behaviour takes place whenever $\beta+\gamma>0$, but there is nonetheless an intrinsic tend- 
ency to hardening [10] if $\gamma<0,|\gamma|<\beta$. It is intuitively clear that the loop shape imposes a different probability distribution for the variable $Z$. In the present research it has been observed that, in fact, the underestimation of the displacement response when using the normality assumption is lower in systems with hardening tendency than in the opposite case.

Obviously, the combination of these errors produce a single discrepancy between Monte Carlo and the analytical results derived with the Gaussian assumption in each specific case. Therefore, the determination of their contributions, with the aim of correcting the Gaussian results with empirical factors, would require an extensive and tedious parametric study.

\section{Non Gaussian linearization approach}

The objective of this section is to develop further the non-Gaussian approach proposed recently by the authors [29]. The method is purported to give better estimations than the Gaussian approach preserving its low computational effort. To such purpose it makes use of mixed

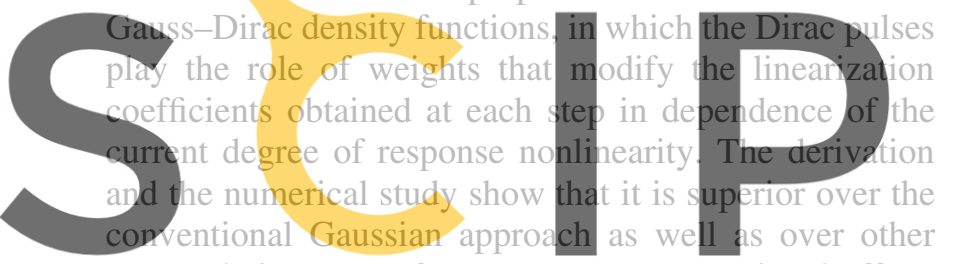
proposals in terms of accuracy or computational effort.

As mentioned above, the concentration of values of the hysteretic restoring force in the vicinity of its maximum $z_{\mathrm{u}}$ suggests the use of a mixed density of the type

$f_{Z}(z)=(1-2 p) \varphi_{Z}(z)+p \delta\left(z-z_{\mathrm{u}}\right)+p \delta\left(z+z_{\mathrm{u}}\right)$

where $p$ is a weighting coefficient. Note that the Dirac pulses play the role of a balancing mass acting on the second statistical moment. Besides, the value of the coefficient $p$ is selected in such a way that it give the best approximation to the Monbe Carlo results in standard cases.

The calculation of the linearization matrices requires the knowledge of joint density functions of the pairs $(X$, $Z$ ) and $(\dot{X}, Z)$. Denoting by $V$ either of $X$ or $\dot{X}$, they are succintly expressed as

$$
\begin{aligned}
& f_{V Z}(v, z)=(1-2 p) \varphi_{V Z}(v, z)+p \delta\left(z-z_{\mathrm{u}}\right) \varphi_{V}(v)+p \delta(z \\
& \left.\quad+z_{\mathrm{u}}\right) \varphi_{V}(v)
\end{aligned}
$$

On the other hand, variables $X$ and $\dot{X}$ can be assumed to remain jointly Gaussian. Note that for separating the contributions of the Gaussian and Dirac parts in calculat- ing the linearization coefficients a dummy splitting of their density functions is needed. i.e.

$f_{V}(v)=(1-2 p) \varphi_{V}(v)+2 p \varphi_{V}(v)$

$f_{X \dot{X}}(x, \dot{x})=(1-2 p) \varphi_{X \dot{X}}(x, \dot{x})+2 p \varphi_{X \dot{X}}(x, \dot{x})$

Note also that the linearity of the above assumed densities and the use of the entire Gaussian function allow the preservation of the Gaussian linearization coefficients, whose closed form is an important prerogative of this kind of models.

\subsection{Second order constraints}

The parameters of the Gaussian density function entering into the linearization coefficients can be enforced to satisfy certain second order constraints. In particular, using Eq. (16), the variance of the hysteretic variable $Z$ is

\section{$E\left[Z^{2}\right]=(1-2 p) \sigma_{Z}^{2}+2 p z_{\mathrm{u}}^{2}$}

(19)

so that the linearization coefficients must be calculated using

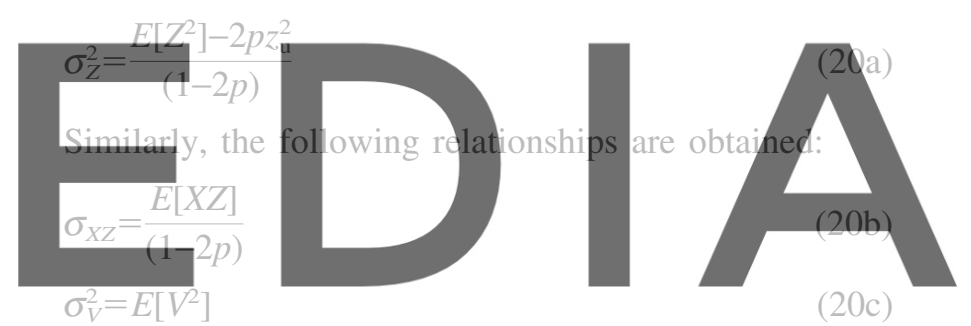

(20c)

\section{down $\bar{n}$ Foad the version without the watermark}

where again $V$ stands for either $X$ or $\dot{X}$ and the expectations are obtained from the solution of the covariance response Eq. (9). Note that for satysfying these consistency requirements when using truncated Gaussian densities as in the approach by Kimura et al. [23], a system of nonlinear equations must be solved at each time step.

\subsection{Linearization coefficients}

The assumed densities Eqs. (14)-(16) lead to the following vector of linearization coefficients [29]:

$H_{\mathrm{e}}^{\mathrm{T}}=\left[s_{\mathrm{e}} c_{\mathrm{e}} k_{\mathrm{e}}\right]=(1-2 p)\left[s_{\mathrm{g}} c_{\mathrm{g}} k_{\mathrm{g}}\right]+2 p\left[s_{\mathrm{d}} c_{\mathrm{d}} k_{\mathrm{d}}\right] \Pi^{-1}$

Here the subindexes "g" and "d" denote Gaussian and Dirac parts, respectively, and in the general MDOF case the $3 \times 3$ matrix $\Pi$ of each degree-of-freedom is built up with the elements of the covariance response $\sum$ :

$$
\Pi=E\left(\begin{array}{ccc}
X^{2} & X \dot{X} & X Z \\
\dot{X} X & \dot{X}^{2} & \dot{X} Z \\
Z X & Z \dot{X} & Z^{2}
\end{array}\right)
$$


The calculation of the Dirac linearization coefficients yields

$s_{\mathrm{d}}=\sigma_{X \dot{X}}\left(A-\gamma z_{\mathrm{u}}^{n}\right)$

$c_{\mathrm{d}}=\sigma_{X}^{2}\left(A-\gamma z_{\mathrm{u}}^{n}\right)$

$k_{\mathrm{d}}=-\sigma_{\dot{X}} \beta z_{\mathrm{u}}^{n+1} \sqrt{\frac{2}{\pi}}$

while those corresponding to the Gaussian part are given by Eqs. (5a), (5b) and (5c); in both parts use must be made of the second order constraints Eqs. (20)a, (20)b, (20)c and (20)d. Note that the new additional coefficients are given in closed form, while their evaluation in the approach using truncated Gaussian functions [23] requires the calculation of several double integrals at each step.

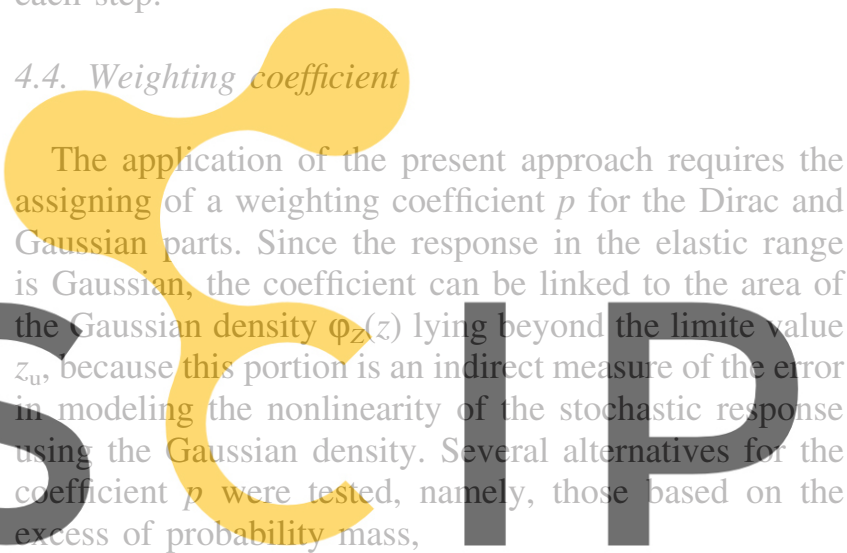

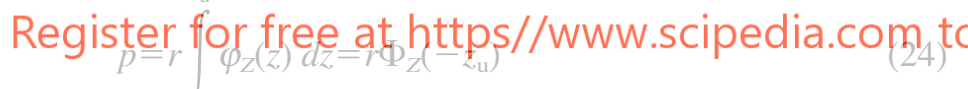

variance, kurtosis and averages of them. After many numerical analysis performed with this algorithm, varying load and model parameters, it was found that the excess-mass criterion was the most stable in the sense that its associated coefficient $r$ was the least influenced by the several conditions imposed on the random vibration of the hysteretic system, such as response level, excitation type, nonstationarity, etc. This coefficient will be discussed in more detail in the following numerical study.

\section{Numerical study}

In order to demonstrate the adequacy of the proposed approach, several numerical analyses were performed on a family of Bouc-Wen oscillators subject to different types of excitations. The system parameters and the loads were varied in order to gain insight into the factors most influential on the errors of the conventional method and the ability of the present approach to improve the estimations.
The structural mass was set equal to unity and no viscous damping was considered. Two very different types of excitations were used, namely white base acceleration with one-sided, constant power spectral density $G_{\mathrm{W}}=288$ $\mathrm{cm}^{2} / \mathrm{s}^{3}$, and the Clough-Penzien double filter with parameters $\omega_{\mathrm{g}}=16.5 \mathrm{rad} / \mathrm{s}, v_{\mathrm{g}}=0.8, \omega_{\mathrm{f}}=2 \mathrm{rad} / \mathrm{s}$ and $v_{\mathrm{f}}=0.65$. Both unmodulated and modulated excitations were considered. The modulating function employed was the same used previously.

For modeling several degrees of nonlinear behavior it was decided to keep constant the excitation intensity and to vary instead the value of the restoring force $h_{\mathrm{u}}$, given by

$h_{\mathrm{u}}=(1-\alpha) k z_{\mathrm{u}}$

To do this $n$ was set equal to unity as well as $A$, in which case $k$ represents the initial stiffness of the system, while the sum $\beta+\gamma$ was varied according to the intended degree of nonlinearity of system's response in the form
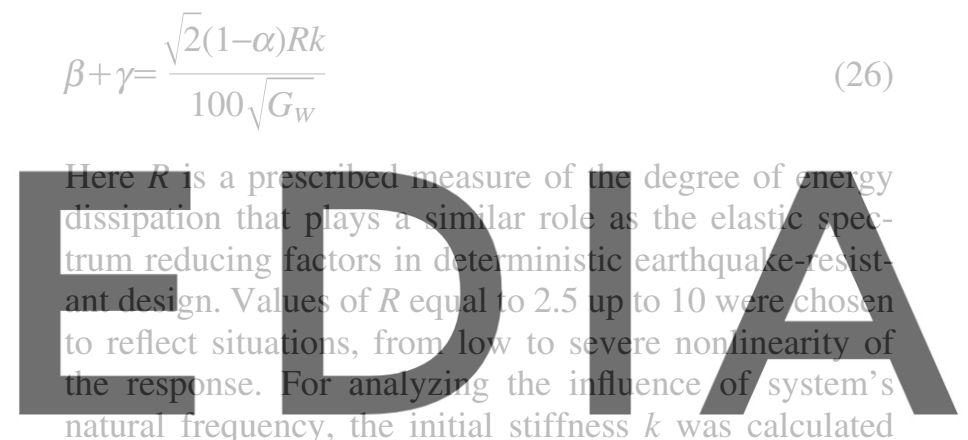

to give the structure initial periods in the range $T=0.25$

downdaadithe, vension nwithouttethenwaterimark

systems without and with hardening tendency. The ratios $\beta / \gamma=1$ and $\beta / \gamma=-2, \gamma<0$ were chosen as representative of such cases.

It is evident that the above experimental design samples adequately the variety of situations in which the Bouc-Wen model is used. The following are the main conclusions drawn from the multiple analyses performed on the two type of cases studied.

\subsection{Softening systems with no hardening tendency}

The most common situation found in the bibliographic survey on the use of the Bouc-Wen hysteretic model corresponds to $\beta+\gamma>0, \beta / \gamma=1$, in which the hardening tendency is completely absent.

The influence of the structural period on the magnitude of errors of the conventional and proposed approaches will be discussed first. Fig. 3 illustrates the response of a middle period system with $R=10$ and $\alpha=0.0$ to white noise. These parameters have been chosen to produce the highest possible error resulting from the Gaussian assumption when the system is subject to white noise, inasmuch as the error is the larger, the stronger the nonlinear behaviour and the lower the value of $\alpha$. A 


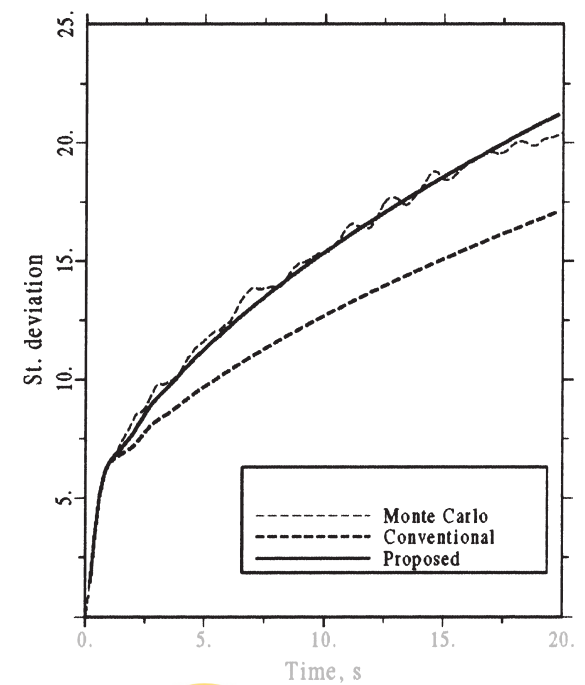

Fig. 3. Displacement response under white noise. $T=1.5, R=10, \alpha=0$. value of $r=-0.5$ was used in Eq. (24), which has been found to be most adequate for this kind of system in the many analyses conducted on this type of system. It can be observed that the proposed approach yields a better S tion. A little improvement velocity and hysteretic function oberved. 4 shows a comparison of $Z$ as postulated by con

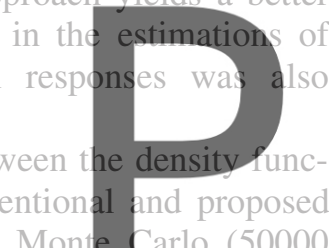

samples). It can be seen that the introduction of the Dirac

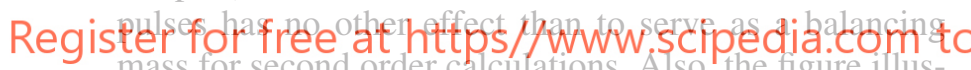
mass for second order calculations. Also, the figure 1 lis as its effect is to flat the peak and raise the tails of the Gaussian function just as much as needed for second

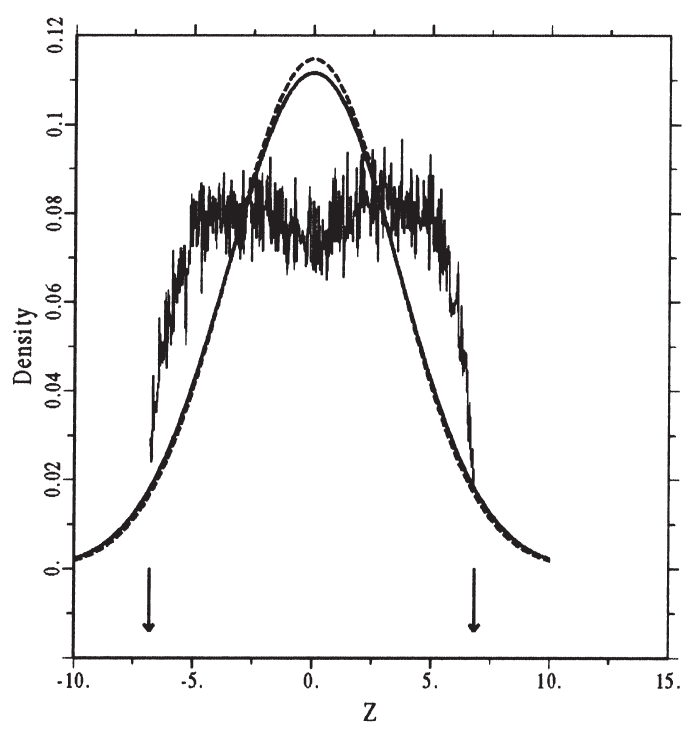

Fig. 4. Density functions of $Z$. order agreement with the empirical density function. Note that such negative $r$ values imply a violation of the strict positiveness required for any density function. However, it must be said that this violation has a minor practical importance for the following reasons: (a) The density functions postulated by the method of equivalent linearization are of little practical use, as they differ from the exact ones in shape and values [25]; (b) Among all the densities postulated by the method, those involving $Z$ (and hence the negative Dirac pulses) would be the least useful in practice for reliability assessment, which is usually performed on the displacement response; (c) The aim of the equivalent linearization method is only to estimate the second order probabilistic response, which allows only a rough assessment of the structural reliability, usually in the form of failure probability bounds, using level crossing theories.

The results corresponding to systems with low and high periods (Fig. 5) indicate that the improvement is achieved independently of the natural frequency. This feature of the proposed approach was observed in many additional analyses as well as the independency of the coefficient $r$ on the post-yielding ratio $\alpha$, which is one

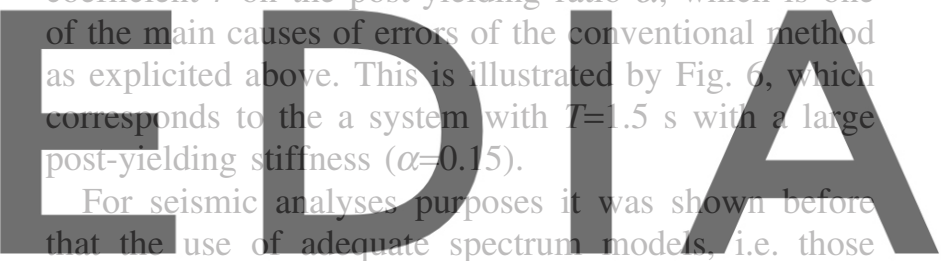

characterized by zero energy at null frequency, makes

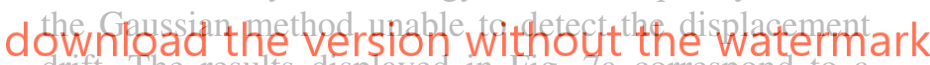
drift. The results displayed in F1g. Ta correspond to a system subject to the Clough-Penzien spectrum with $\alpha=0$. A period $T=0.4 \mathrm{~s}$ was used in order to subject the system to a nearly-to-resonance state. The superiority of the proposed approach in this important respect is evident, as it is able to detect the presence of drift. However, it can be observed that the start of the correcting effect of the Dirac pulses takes place later than the somewhat abrupt switching of the drift errance. As a result, the drift path of the Monte Carlo and proposed estimations are different in shape. Nevertheless, the delay is much less pronounced when smooth amplitude modulating functions are used, as is common practice in seismic random vibration analysis. This is illustrated by Fig. $7 b$, which adds to the results depicted in Fig. 2 the estimation afforded by the proposed method. It can be observed that the error of the proposed approach is negligible for practical purposes.

It should be observed that the proposed approach can follow the drift path just due to the introduction of the Dirac pulses. As it was pointed out before, under Gaussian input the response of a linear system is always Gaussian. This implies that an indirect way of compensating for an effect of linearization, namely the supres- 
(a)

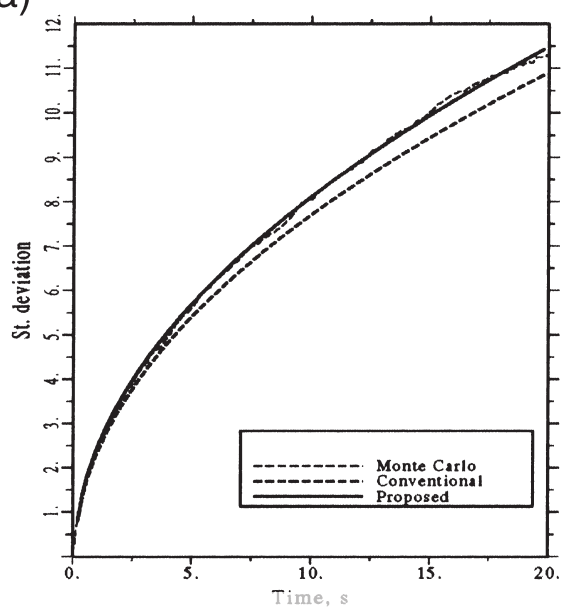

(b)

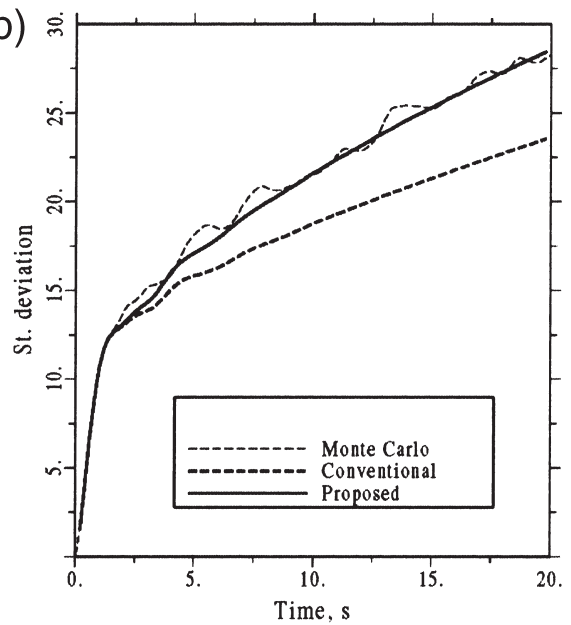

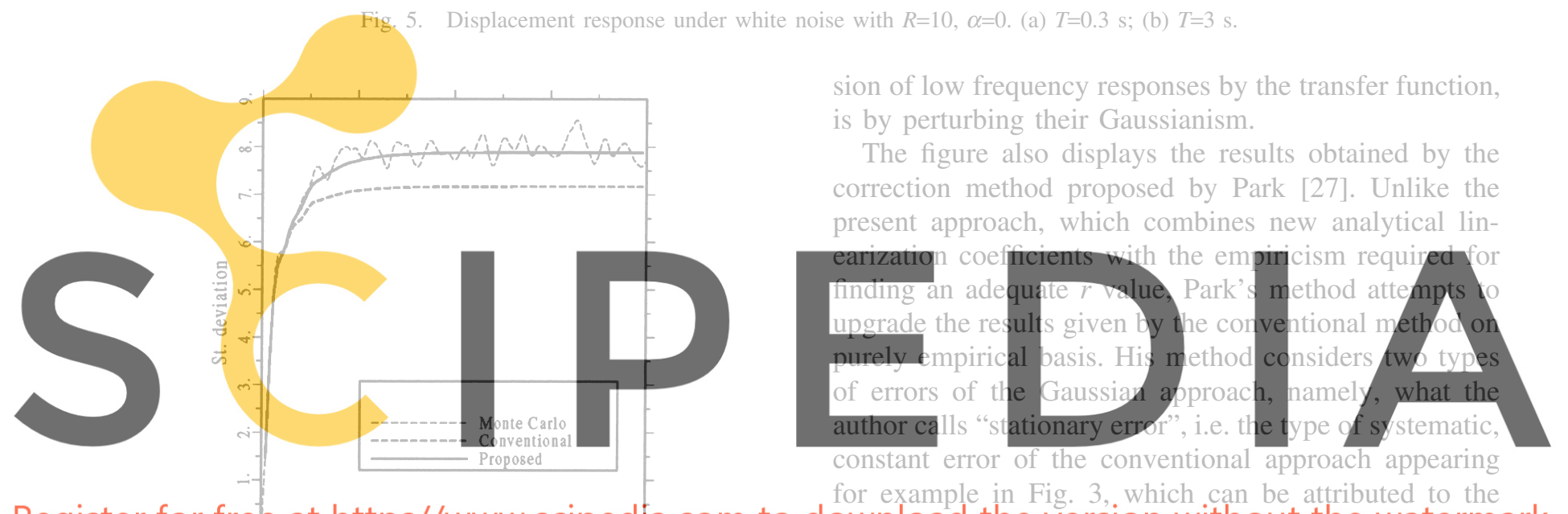

Register for free at https//www.scipedia.com to dowsindaad theidessioprobaithoutetheewater rmark

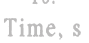

Fig. 6. Displacement response under white noise. $T=1.5, R=10$, $\alpha=0.15$. second, "nonstationary" error, which corresponds to drift. It must be observed that the coefficients have been empirically derived for the rather specific situation of a Iwan-Paparizos [24] seismic spectrum, a specific modul- (a)

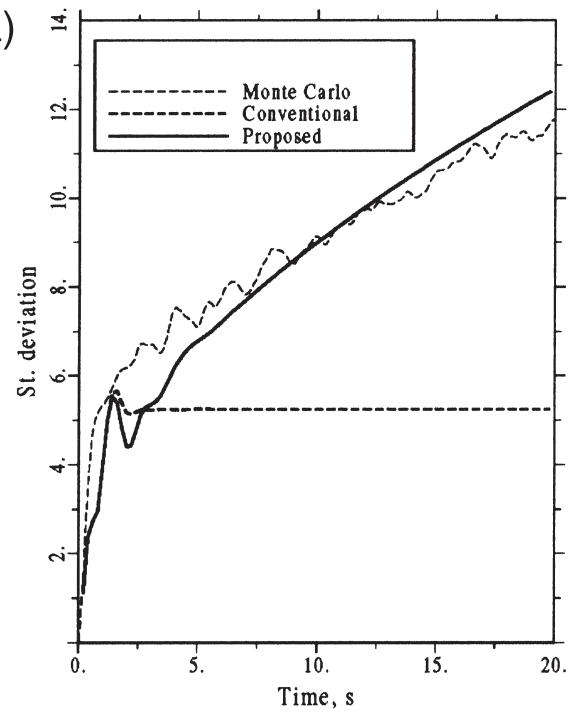

(b)

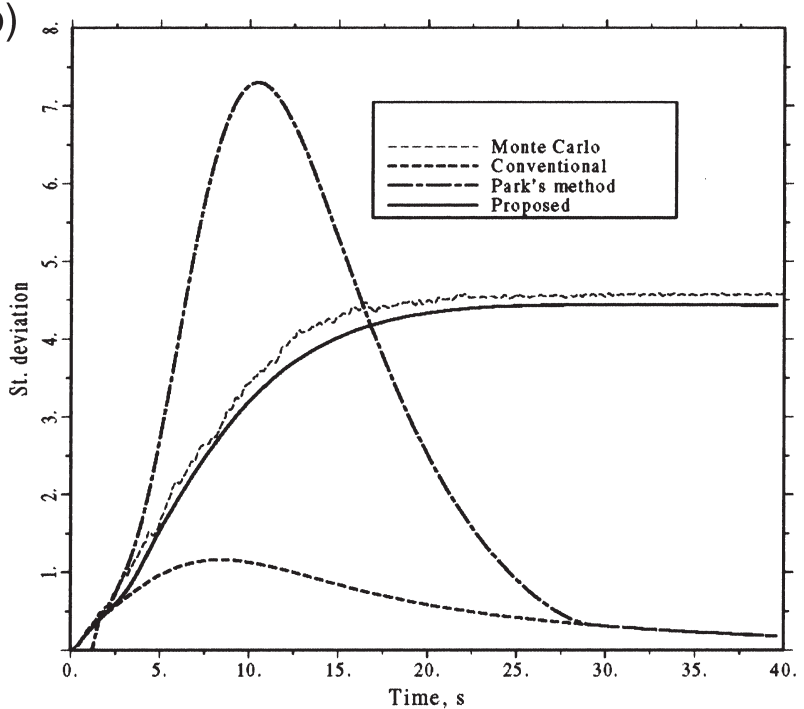

Fig. 7. Displacement response under Clough-Penzien excitation. $T=0.4, \alpha=0$. (a) Unmodulated; (b) Modulated. 
ating function and an initial frequency equal to that corresponding to the spectral peak. As a consequence, the empirical method is expected to give erroneous estimations in a different situation, such as that of Fig. 7b. In fact, the peak of the standard deviation of the displacement is largely overestimated. Moreover, insofar as Park's method attempts to modify a posteriori the results given by the Gaussian method by a multiplying factor, the erroneous shape of the latter is maintained.

As it was said before, the errors of the Gaussian method diminish when the post-yield ratio $\alpha$ increases due to the implied reduction of drift. This is illustrated by Fig. 8 , which corresponds to a system with $\omega_{\mathrm{g}}=16.9$ $\mathrm{rad} / \mathrm{s}, \nu_{\mathrm{g}}=0.94$ and $\alpha=0.05$. The estimation given by Park's method in this case are also far from the Monte Carlo results, while the agreement of the proposed approach with the Monte Carlo analysis is again excellent.

The cases analysed so far correspond to large incursions in the nonlinear range, as reflected by the factor $R=10$. For lower degrees of nonlinear behaviour less concentration of $Z$ values near its maximum and hence a different probability density of $Z$ can be expected. As
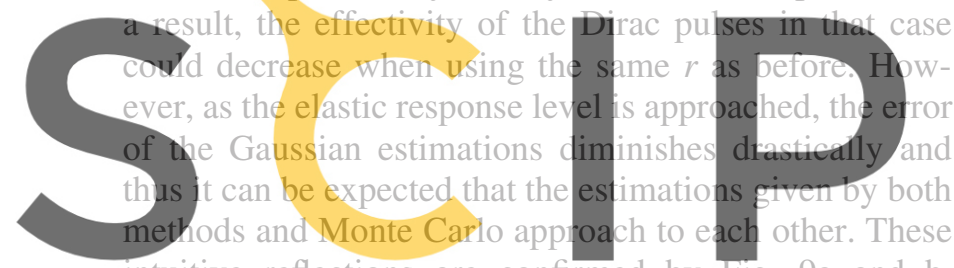

intuitive reflections are confirmed by Fig. 9a and b, which correspond to $R=5$ and $R=2.5$ respectively. A per-

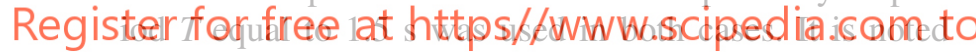
that in the first case the same $r=-0.5$ used before leads

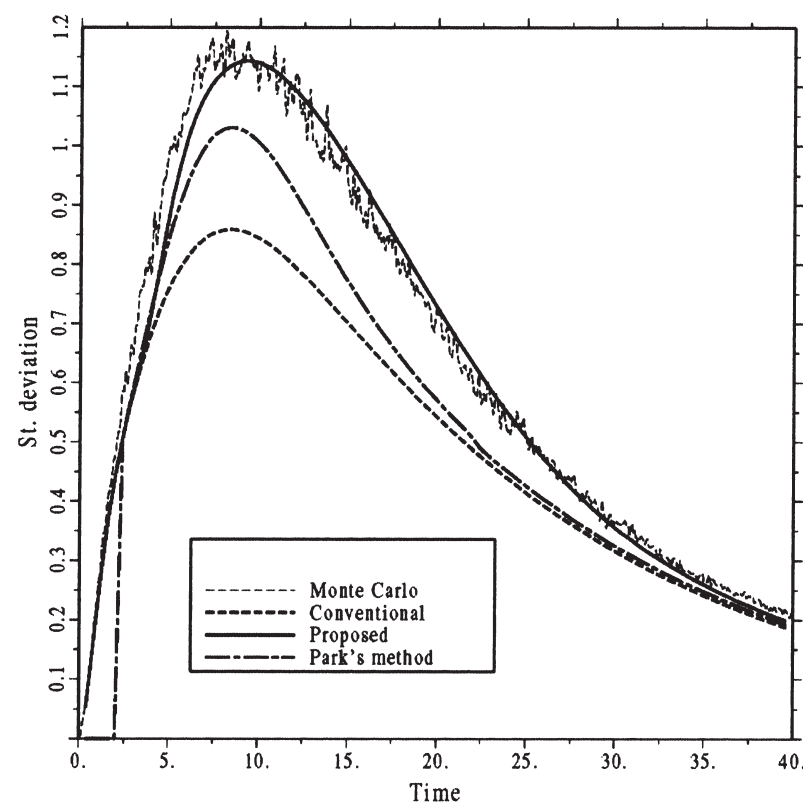

Fig. 8. Displacement response under modulated Clough-Penzien load. $\alpha=0.05$. to estimations which are somewhat, unconservative but anyway better than those stemming from the assumption of Gaussianity. On the other hand, it can be seen that the estimations given by both methods approach those of Monte Carlo simulation as $R$ decreases. The figures also display the results obtained using $r=-0.8$ and $r=-11$, respectively, which are closer to the exact ones. In general, the empirical curve for the $-r$ coefficient depicted in Fig. 10 can be used for obtaining more accurate estimations of the displacement response of this type of system for low degrees of nonlinear behaviour. The coefficient has been put in relation to a sort of ductility ratio given by

$a=\frac{\sigma_{0.5}}{x_{\mathrm{y}}}$

where $x_{\mathrm{y}}$ is the yield displacement of the system and $\sigma_{0.5}$ is the standard deviation of the displacement obtained with $r=-0.5$ at $t=20 \mathrm{~s}$ of response when subject to unmodulated white noise. The dots displayed in the figure were obtained by searching the $r$ value giving the closest results to the statistics of 1000 Monte Carlo simulations. The equation of the empirical curve is

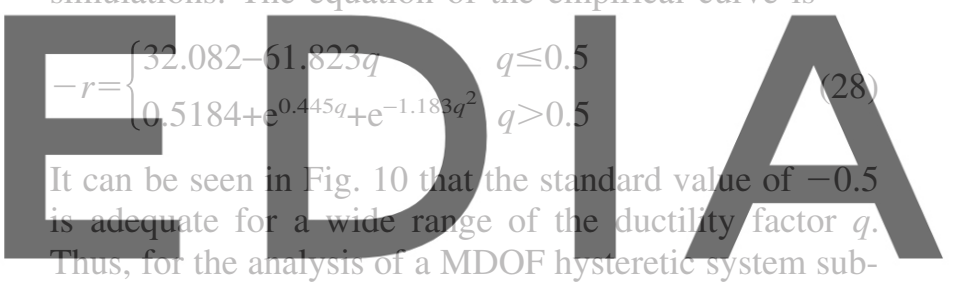

ject to random excitations, this value can be applied to

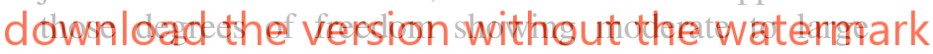
energy dissipation. For those showing small incursions in the nonlinear range there would be a need of analysing the structure using the reference value in order to calculate an upgraded one by means of Eq. (28). However, complications can be avoided if use is made of the standard value of -0.5 for the entire structure, due to the following reasons: (a) Such degrees of freedom are of lower importance insofar as their contribution to the overall probability of failure is small; (b) As illustrated by Fig. 9, the underestimation of the displacement with $r=-0.5$ is slight in percent when compared to that ocurring at high levels of response nonlinearity.

The method has been included in a general equivalent linearization code for MDOF systems. No computational problems have been observed in the numerous MDOF test analyses performed.

\subsection{Softening systems with hardening tendency}

As said in the preceeding, a negative $\gamma$, such that $\beta+\gamma>0$ (softening condition) and $|\gamma|<\beta$ imposes a tendency to hardening. Consequently, the frequency distribution of $Z$ will be different from those corresponding to $\gamma>0$ and hence this fact should have a reflect on the 
(a)

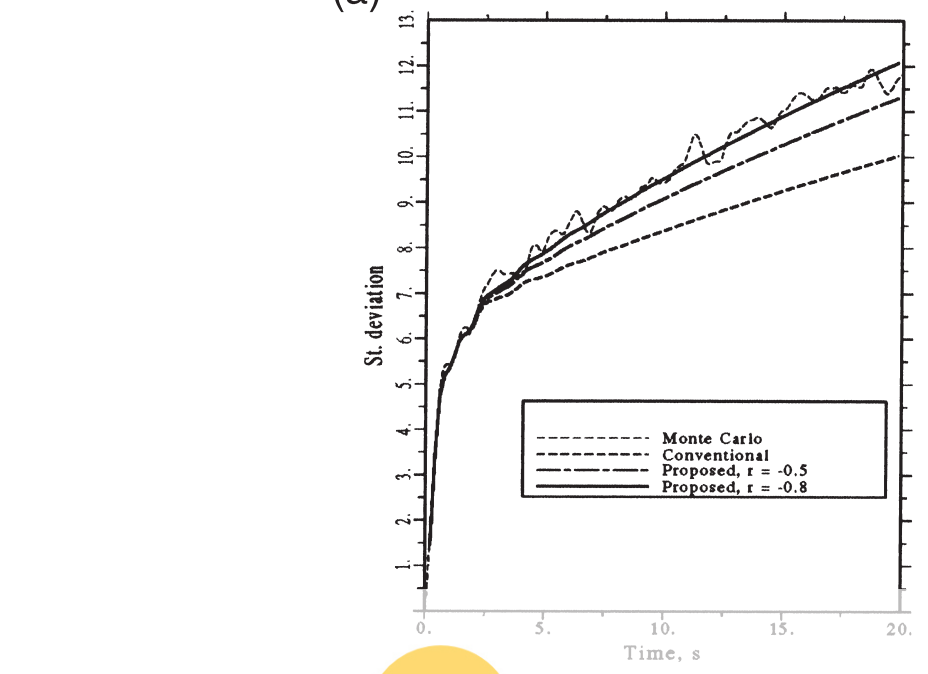

(b)

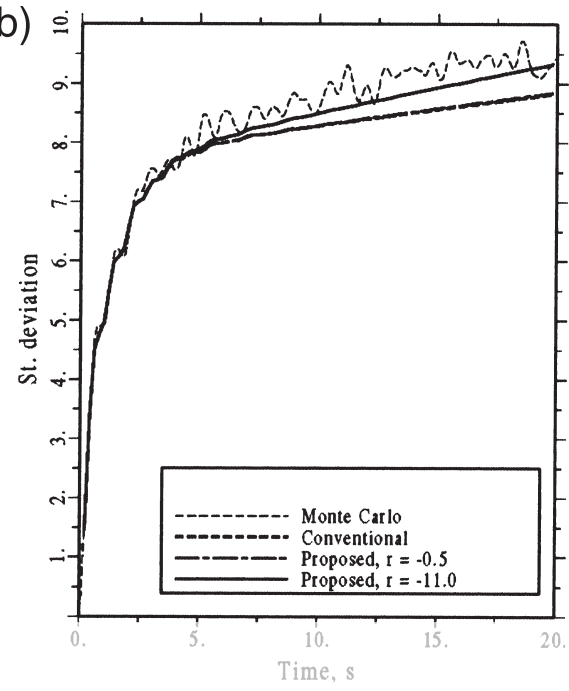

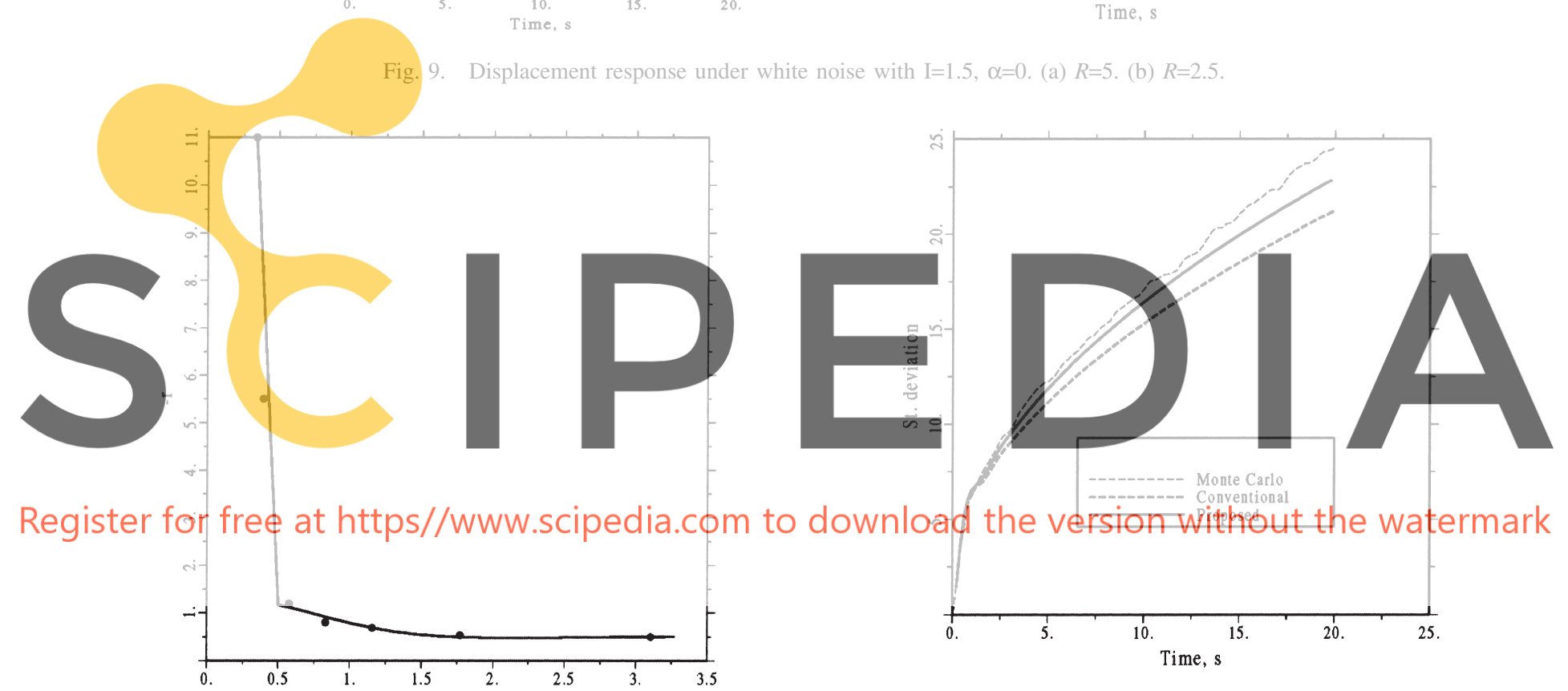

Fig. 11. Displacement response of system with $\beta / \rho=-2$ under white noise. $T=1.5, R=10, \alpha=0$.

Fig. 10. Empirical curve of $-r$ for $\beta / \gamma=1$.

selection of parameter $r$. As an example, let us consider the case $\beta / \gamma=-2, \gamma<0$. Fig. 11 shows the results corresponding to $R=10$ for a structure having $T=1.5$ and $\alpha=0$ subject to white noise excitation calculated with $r=-0.22$, which appeared to be suitable to this type of system. The agreement of the proposed approach with the simulation results is better in Fig. 12, which corresponds to a system akin to that of Fig. 10 with $\beta / \gamma=-2$. No regression curve for $r$ has been calculated in this case.

\section{Conclusions}

The following conclusions can be drawn from the above research:
1. The conventional method, which makes use of the hypothesis of Gaussian behaviour for all the state variables, due to the important simplifications resulting from such an assumption, leads to errors in the estimation of the structural displacements. The magnitude of these errors can be as high as $300 \%$ in practical earthquake engineering cases.

2. The main causes of the errors are: (a) The Gaussian density assumption as such, which is theoretically exact only in case of linear systems driven by Gaussian inputs. (b) The drift trend of hysteretic systems, which is especially conspicuous for those having a very low post-yield ratio. (c) The filtering of low frequencies in spectral seismic models, which precludes the possibility of reproducing the drift contribution to the displacement response. (d) The shape of the 


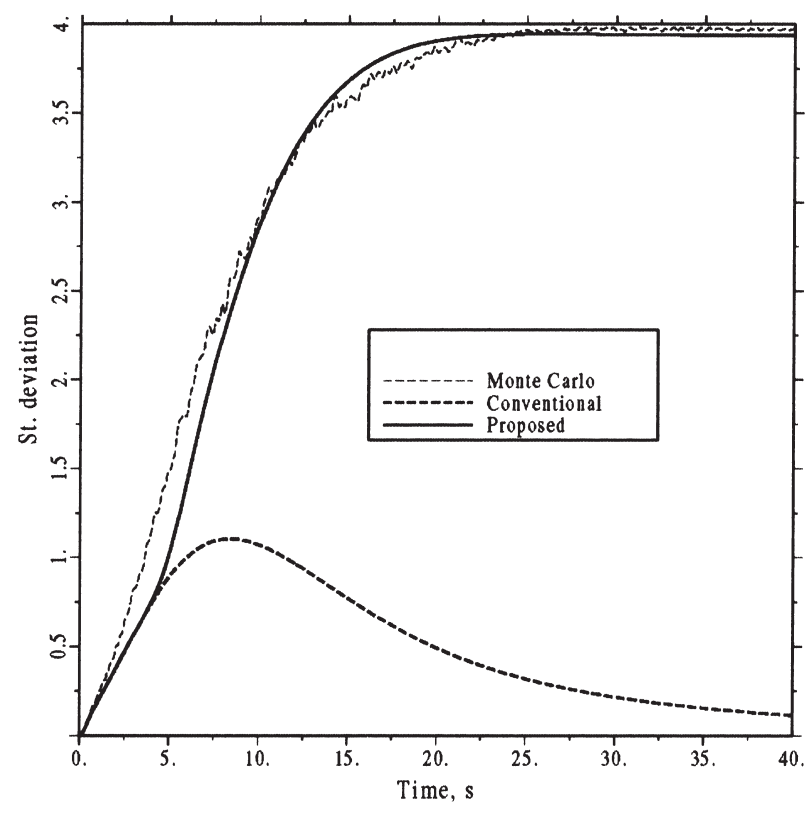

Fig. 12. Displacement response of system with $\beta / \gamma=-2$ under modulated Clough-Penzien excitation.

hysteretic loops, which influences the actual probabilistic distribution of the restoring force.

3. The application of the proposed technique, based on a combination of Dirac and Gauss densities, depends on an empirical coefficient that must be callibrated through Monte Carlo simulation for each combination of the pair $(n, \beta / \gamma)$ on SDOF systems. In the present research, the values -0.5 and -0.22 have been found adequate for the cases $(1,1)$ and $(1,-2)$, respectively, which are widely used.

4. The empirical parameter could be corrected for low degrees of response nonlinearity in order to obtain better estimations. A regression curve for the case $(1,1)$ is proposed. However, the recalculation of the response can be avoided if considering that in such cases the errors when using the standard value of the empirical coefficient are low.

5. The numerical analyses demonstrate that the proposed approach represents an important improvement of the method of stochastic linearization when applied to hysteretic systems of the softening type, because its estimations of the response statistics are in better agreement with Monte Carlo simulation than those given by the conventional Gaussian technique. In particular, its ability to detect the drift motion characteristic of this type of systems allows to obtain closeto-exact solutions while the conventional method fails with large errors.

6. The proposed approach has distinct advantages over alternative proposals with similar aims. Particularly, it is computationally as simple as the classical Gaussian approach, because its linearization coefficients have a closed form as well. In contrast, other approaches based upon transformations require the solution of nonlinear systems and multiple integrals at each step of the solution and/or the realization of some Monte Carlo simulations for obtaining improved density functions. Also, the proposed method is more accurate than an empirical technique proposed to upgrade the estimations obtained with the Gaussian classical method.

7. The negative $r$ values imply a violation of a fundamental condition by the postulated probability densities involving the hysteretic variable $Z$. However, these and other density functions postulated by the method are of little practical value due to their poor accuracy. In fact, the calculation of better estimates of the densities lies beyond the scope of the stochastic linearization method, which is only purported to estimating second order responses.

\section{Acknowledgements}

Financial supports for the realization of the present research has been received from the Colombian Science Institute, Colciencias, and the National University of Colombia. The work has also been partially supported by the ESPRIT programme of the Comision of European Countries, project PROMENVIR, ESPRIT 20189, and by the "Dirección General de Investigación Científica y Técnica" (DGICYT) of the Spanish Government under the Grant No. PB93-1040. The supports are gratefully acknowledged.

\section{References}

[1] Krylov N, Bogoliubov N. Introduction to nonlinear mechanics. New York: Princenton University Press, 1943.

[2] Caughey TK. Equivalent linearization techniques. J Acoustical Soc Am 1963;35:1706-11.

[3] Iwan WD. A generalization of the concept of equivalent linearization. Int J Nonlinear Mech 1973;8:279-87.

[4] Atalik TS, Utku S. Stochastic linearization of multi-degree of freedom non-linear systems. Earthquake Engng Struct Dynamics 1976;4:411-20.

[5] Faravelli L, Casciati F, Singh MP. Stochastic equivalent linearization algoritms and their applicability to hysteretic systems. Meccanica 1988;23:107-12.

[6] Spanos PD, Iwan WD. On the existence and uniqueness of solution generated by equivalent linearization. Int $\mathrm{J}$ Non-Linear Mech 1978;13:71-8.

[7] Roberts JB, Spanos PD. Random vibration and statistical linearization. Chichester: John Wiley and Sons, 1990.

[8] Bouc R. Forced vibration of mechanical systems with hysteresis (Abstract). In: Proceedings of the Fourth Conference on Nonlinear Oscillation, Prague, 1967.

[9] Wen YK. Approximate method for non-linear random vibration. J Engng Mech 1975;101:389-401.

[10] Baber TT, Wen YK. Random vibration of hysteretic, degrading systems. J Engng Mech Div ASCE 1981;107:1069-87. 
[11] Casciatti F, Faravelli L. Methods of non-linear stochastic dynamics for the assessment of structural fragility. Nuclear Engng Des 1985;90:341-56.

[12] Foliente GC, Singh MP, Noori MN. Equivalent linearization of generally pinching hysteretic, degrading systems. Earthquake Engng Struct Dynamics 1996;25:611-29.

[13] Cunha AAMF. The role of the stochastic equivalent linearization method in the analysis of the non-linear seismic response of building structures. Earthquake Engng Struct Dynamics 1994;23:837-57.

[14] Sues RH, Wen YK, Ang AHS. Stochastic evaluation of seismic structural performance. J Struct Engng 1985;111:1204-18.

[15] Sues RH, Mau ST, Wen YK. System identification of degrading hysteretic restoring forces. J Engng Mech 1988;114:833-46.

[16] Wen YK, Eliopoulos D. Method for nonstationary random vibration of inelastic structures. Probabilistic Engng Mech 1994;9:115-23.

[17] Pires JEA, Wen YK, Ang AHS. Stochastic analysis of liquefaction under earthquake loading. Civil Engineering Studies, Report SRS No. 504, University of Illinois at Urbana-Champaign, 1983.

[18] Casciatti F, Faravelli L. Stochastic equivalent linearization for 3D frames. J Engng Mech ASCE 1989;114:1760-71.

[19] Lin BC, Tadjbakhsh IG, Papageorgiu AS, Ahmadi G. Response of base-isolated building to random excitations described by the Clough-Penzien spectral model. Earthquake Engng Struct Dynamics 1989;18:49-62.

[20] Yang JN, Li Z, Vongchavalitkul S. Stochastic hybrid control of hysteretic structures. Probabilistic Engng Mech 1994;9:125-33.

[21] Li Z, Katsukura H. Markovian hysteretic characteristics. J Engng Mech ASCE 1990;116:1798-811.
[22] Kobori T, Minai R, Suzuki Y. Stochastic response of hysteretic structures. Bulletin of the Disaster Prevention Research Institute. Kyoto University 1977;26:57-70.

[23] Kimura K, Yasumuro H, Sakata M. Non-gaussian equivalent linearization for nonstationary random vibration of hysteretic system. Probabilistic Engng Mech 1994;9:15-22.

[24] Iwan WD, Paparizos LG. The stochastic response of strongly yielding systems. Probabilistic Engng Mech 1988;3:75-82.

[25] Pradlwarter HJ, Schuëller GI. The method of statistical equivalent linearization. In: Schueller GI, editor, Structural Dynamics Recent Advances. Berlin: Springer-Verlag, 1991.

[26] Nataf A. Determination des distributions dont les marges sont donées. Comptes rendues de l'Acaademie des Sciences 1962;225:42-3.

[27] Park YJ. Equivalent linearization for seismic responses. I: Formulation and error analysis. J Engng Mech ASCE 1992;118:220726.

[28] Yell CH. Modeling of nonstationary earthquake ground motion and biaxial and torsional response of inelastic structures. Ph. D. thesis, University of Illinois, 1989.

[29] Hurtado JE, Barbat AH. Improved stochastic linearization method using mixed distributions. Struct Safety 1996;18:49-62.

[30] Clough RW, Penzien J. Dynamics of structures. 2nd ed. New York: McGraw Hill, 1993.

[31] Shinozuka M, Sato Y. Simulation of nonstationary random processes. J Engng Mech Div 1967;93:11-40.

[32] Yang JN, Liu SC. Distribution of maximum and statistical response spectra. J Engng Mech Div 1981;107:1089-102. 